\title{
The Public User's Role in Breast Self-Examination Teleconsultation System Development: Modified User-Created Content Approach
}

\author{
${ }^{1}$ Norayu A. Talib, ${ }^{1,2}$ Nasriah Zakaria, \\ ${ }^{1}$ Sureswaran Ramadass and ${ }^{2}$ Faten Damanhoori \\ ${ }^{1}$ National Advanced IPv6 Centre of Excellence (NAv6), School of Computer Sciences, \\ University Sains Malaysia (USM), 11800 Penang, Malaysia \\ ${ }^{2}$ School of Computer Sciences, University Sains Malaysia (USM), 11800 Penang, Malaysia
}

\begin{abstract}
Problem statement: Teleconsultation services had been introduced in Malaysia for almost 10 years yet it has not been optimally adopted even though it was found that teleconsultation in Malaysia is cost saving, efficient in allocating resources, enhances diagnostic options and better health outcomes. At present, the underutilized service is due to its failure to satisfy the user's demands on various aspects. Since Teleconsultation in BSE is at proposal stage, there is strong need for user's involvement in suggesting its contents to harvest end-user acceptance. This study describes the development process of the Web-based Breast Self-Examination (BSE) Teleconsultation System, emphasizing the role of public users in identifying the contents of the system and its functionality using our proposed Modified UCC (MUCC) approach. To help understanding the process we first presents the key concepts related to original User-Created Content (UCC) approach; next we describes in details the data gathering process which applied qualitative method; we then describes the web-based application for BSE Teleconsultation, as well as, the development process illustrating the transition from the MUCC to the system development; and finally we discusses the importance of the user's input in the system development from our experience. Approach: A semi-structured one-to-one interviews with 15 public users was carried out in October 2008 in one of the public university in Malaysia to capture the content of the system based on our proposed MUCC approach. Results: By using our MUCC approach, the content of the proposed system has been identified and the BSE Teleconsultation system has been successfully developed. Conclusion/Recommendations: MUCC approach proposed and used in this study has provides insights on user's role in e-health system development.
\end{abstract}

Key words: Breast Self-Examination (BSE), User-Created Content (UCC), teleconsultation system, qualitative method, Modified UCC (MUCC), Requirement Engineering (RE), Social Networking Sites (SNS), Object Oriented Analysis and Design (OOAD)

\section{INTRODUCTION}

The user's role in system development has found to be a significant factor that improves the success of any information system implementation (Schaper and Pervan, 2007). Incorporating user in system development is found in user-centered approach, Requirement Engineering (RE) approach and user-created content (UCC) approach. However, the question arises on how UCC can be applied for e-Health website since this approach has been widely implemented in its application to Social Networking Sites (SNS) (Chin, 2006).

Teleconsultation services had been introduced in Malaysia for almost 10 years yet it has not been optimally adopted even though it was found that teleconsultation in Malaysia is cost saving, efficient in allocating resources, enhances diagnostic options and better health outcomes (Maarop et al., 2009). Thus, we are aware of some issues when proposing teleconsultation as the communication medium for disseminating BSE technique to public. One of the major issues is how to increase the acceptance and adoption of such system so that it will be utilized in the future after it is introduce to the public.

Moreover, we know that many women are now concerning about their health and seeking for advice and supported information on the internet (Seals et al., 2008). This may imply a high potential of women's acceptance of the BSE Teleconsultation as well (Talib et al., 2009). In line with what was suggested by Lannin and Ponn (2005), a personalized BSE Teleconsultation system that delivers various BSE educational messages 
based on public user's (women) suggested contents is designed and developed.

This study describes the development process of BSE Teleconsultation system, emphasizing the role of the users in creating the contents of the system and its functionality using the UCC approach. To present this subject, this study is structured in four parts: (i) a brief description of the key concepts related to original usercreated content (UCC) approach; (ii) a section describing the material and methodology of data gathering process; (iii) a section describing the webbased application for BSE Teleconsultation, as well as, the development process; and finally, (iv) a brief discussions on our experience applying approach in developing the system.

\section{Background: User-Created Content (UCC)} Approach: Incorporating user in design process has applied in Social Networking Sites (SNS) and corporate intranet website (Chin, 2006). The success of any implemented information system depends on how well it fits the users' needs (Davis, 1993). In order to develop system that fulfills the abovementioned requirements, user involvement is essential in determining their needs.

User-created content (UCC) content (UGC) approach is originated in 1990s (Ryu et al., 2009) and can be defined as content created by users themselves (Chin, 2006). UCC has found to be the important factor contributes to the acceptance of some of the most relevant and fastest applications available on the web (Ryu et al., 2009).

The three ways in which UCC can be applied is described as follows based on (Chin, 2006).

Pre-production mode: is where the content is made available only after it is peer-reviewed and verified by knowledge experts.

Post-production mode: is where the content is immediately available the moment it is posted by user. The moderator or knowledge expert has the authority to review the live content and make necessary changes to it based on site's policy.

Peer-based mode: is where the content is made available immediately the moment it is posted by user without moderated by knowledge experts.

UCC incorporating user perspectives into the system development process to fit expectations. Some relevant evidence found in the literature shows that incorporating the UCC approach in software development process increase usage as listed in Table 1 as follows.

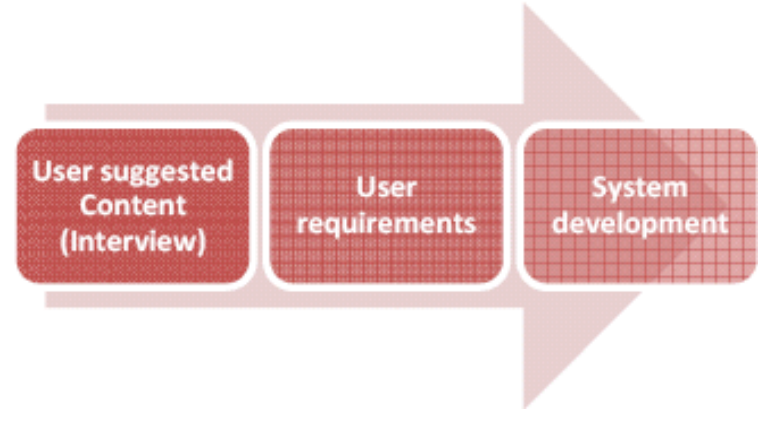

Fig. 1: Modified UCC

Table 1: Relevant literature on UCC approach

\begin{tabular}{ll}
\hline Author & Application \\
\hline (Ryu et al., 2009) & Video UCC \\
(Lee and Jo, 2009) & Bidirectional TPEG \\
(No and Rhee, 2010) & Mobile based SNS \\
(Kim and Jeong, 2009) & Social e-learning website \\
(Lee et al., 2007) & VR@ home \\
(Hjorth, 2009) & Web U2 \\
\hline
\end{tabular}

Despite of UCC benefits, Chin (2006) identified UCC drawbacks associated with: (1) contents overlapping or duplicate, (2) credibility issues of the suggested content, (3) questionable or biased content and (4) the difficulty in organizing the contents since it's structure-free.

Due to this problem, question arises on how this approach can be applied in e-health website/system where the credibility and integrity issues are at stake (Ahern et al., 2006). Therefore, we have modified the original UCC approach suggested by Chin (2006) to overcome the abovementioned problems and to fit its application on web-based health-related system.

In this study, the modified UCC can be defined as the content and functionality of health-related sites/system suggested by user that is peer-reviewed and verified by system developer based on its relevance during the system development process. This approach is differing from the original UCC approach since it is applied during the system development process whereas in original UCC, the suggested content is specifically for already implemented sites/system. The Modified UCC (MUCC) approach applied in this study is illustrated in Fig. 1.

\section{MATERIALS AND METHODS}

Preliminary Study: The study was conducted at a period when no BSE Teleconsultation System prototype was available to be presented to research participants. The aims were therefore to derive the contents of the BSE Teleconsultation system suggested by the public 
user. Thus, contents were elicited through semistructured interviews. Interview is a valuable qualitative data collection method that provides deep understanding of the situations being scrutinized (Voida et al., 2004; Bogdan and Biklen, 1998).

Data were obtained from semi-structured interviews that were audio taped and were conducted in October 2008. The interviews lasted between 25-30 min. The participants were interviewed at one of the public university in Malaysia.

To enhance consistency and reliability of the interviews, an actual interview guide for the study was developed after extensive literature review which involved reviewing related journals. The guide presents brief scenarios to illustrate the BSE Teleconsultation system, followed by a series of questions aimed at determining the contents of the system and its functionality. An informed consent was given to the participant. An Incentive was given as a token of appreciation for respondents' participation in the study.

Emerging themes were explored in interviews with subsequent participants. Audiotapes of the interviews were later transcribed and analyzed qualitatively. 15 transcripts were read independently and a coding frame was developed for the analysis. All transcripts were coded independently using ATLAS.ti. ATLAS.ti aided in managing all the identified codes and quotations from the coded interviews. The programme also shortened the analysis time when compared to the time needed for manual coding (Friese, 2011).

The data analysis process involved four stages, which is based on (Bogdan and Biklen, 1998): (a) Reading all the material to obtain an overall impression; (b) Identifying units of meaning (develop coding categories), representing different types of possible BSE Teleconsultation systems' content required by public users; (c) Condensing and summarizing the contents of each of the coded groups and (d) Summarizing the contents of each code group to generalize descriptions and concepts based on the aims of the study.

Since Malaysia consists of multi-culture citizens, the quotes were spoken in bilingual (mixture of Malay language and English). Therefore, quotes from the interviews were translated from Malay language to English language. The translation is carried out and validated before being manipulated during data analysis.

\section{RESULTS}

From the interview, the participants suggested contents of the BSE Teleconsultation System. Quotes corresponding to the contents suggested by the participants are explained in detailed in this section.
From the interview, the data gathered is transformed into user requirements which help in designing the BSE Teleconsultation system based on public users' input. This method has been previously applied in (Morris and Dillon, 1997; Hughes, 2009).

Forum: The Forum/Discussion is created to enable users to create a topic/thread and reply to existing thread entries. Users may add new topic to the discussion panel. Users will also be able to reply to existing thread entries. New entries will automatically updated after user hit the "post" button. The quotation corresponding to forum is as follow:

- $\quad$ "For a forum, I think it is a good platform for us to share our thoughts and make out friends. Who knows, one day if we have some trouble and does not know where to seek for help we can turn to them."(P7, 20 years old)

Reminder alert: Reminder is created to enable an email reminder to be sent to users 2-hours before their teleconsultation session so that they will be notified and do not forget their pre-booked session. The quotation corresponding to reminder alert is as follow:

- "But they don't have reminder system where we can personalize our monthly reminder to do BSE. I would like to have a feature like that if possible. Then, the alert message can be sent via email or SMS or in personalize page."(P7, 20 years old)

Breast cancer survivor: This module is for breast cancer survivor to share their experience fighting and surviving cancer. The quotation corresponding to breast cancer survivor is as follow:

- "Breast cancer survivor section might help us to see what's exactly and how they recover from breast cancer. Those survivors, spouse or their family members can share their experience in fighting cancer. Their experience may help others who might have facing the same problem. This is a good platform to share the experience and grief. For those survivors, they can give advice and on the other hand will create more aware of this disease especially for those who do not care much about breast care and breast cancer. Maybe after they read the true confession of these survivors, they will have more awareness and go for learning BSE as one of the prevention solution." (P7, 20 years old) 
Breast cancer info/prevention: This module is just a category of breast cancer related information. The contents are subject to change based on demands. These contents were suggested by public users during preliminary study (explained in earlier section). The quotation corresponding to breast cancer related information is as follow:

- "I think a portal should have all the information regarding breast cancer" (P6, 21 years old)

Online appointment booking: This module is for appointment booking. Once the user successfully selects and books an appointment, list of appointments and status is displayed on the personal appointment panel. Users need to wait for an approval within 2 days that will be sent by the selected clinic. The quotation corresponding to online booking (appointment) is as follow:

- "...it is great to have this kind of functionality as we can book the session online." (P7, 20 years old)

Dietary information: This module is created to display all the information about food for breast cancer prevention. The quotation corresponding to dietary information is as follow:

- “...it's like nutrition also.....for example a proper diet how to avoid breast cancer." (P10, 19 years old)

Live chat (video and audio): The chat module enables user to have community chat among users. It enhances communication among users.

This module enables users to have one-to-one consultation with a nurse/doctor after an appointment has been approved. Users can chat personally with the nurse/doctor while having teleconsultation session. This service can be customized based on users' preferences because it has functionalities such as: (1) Turn on/off the camera, (2) Turn on/off the speaker (voice), (3) Hands free and (4) Broadcast video functionality.

The broadcast video function can be enabled or disabled, depending on users' preferences (either to allow video broadcasting or only for the intended person). The quotation corresponding to forum is as follow:

- "I think it would be nice if we have chat function like live chat or forum where we can join a thread." (P6, 21 years old)

Testimonial: Testimonial module enable user to create or add testimonial entry. This function enables current users to share their thoughts and experience of using the system. Users may add new testimonials to the testimonial panel. The quotation corresponding to testimonial is as follow:

- "You can share your experience when you have tried the system...maybe can give inspiration to other people to try and use the system..." (P5, 20 years old)

Personalized page: This module is for displaying all the information related to user. It is also display the appointment details and reminder details. The quotation corresponding to personalized page is as follow:

- $\quad$ I imagine having something like personalized page...where we can upload our personal details and when we booked the appointment, the appointment details will be displayed in our page...so it will be easy for us to remember. Then any alert we will have it in our personalized page.” (P6, 21 years old)

Other contents: Download section and Hospital directory. This module is for download functionality where user may download resources. Hospital directory will listed all the participating hospital or clinic. The quotation corresponding to download section and hospital directory are as follow:

- “....any downloadable materials like video or pamphlet and any important information like where to get help." (P6, 21 years old)

- "Maybe information of clinics and hospitals...list of clinics....contact number, address and contact person...so that if we need further assistance, we can refer to the list." ( $\mathrm{P} 4,22$ years old)

The BSE teleconsultation system In this section we will describe briefly on the BSE Teleconsultation system (Talib et al., 2008). Then we presents the main techniques and methods used in the development process, as well as the main result obtained.

System overview: The BSE Teleconsultation system prototype can be described as three-tiered client-server architecture (Burback, 1998). Client in the architecture 
implemented is a thin-client (Lowber, 2002). Therefore, the presentation service is just a web browser. Process service (application service) is the interface between the presentation service (web browser) and the data (database). In this system they are implemented using ASP.Net, Visual Basic.Net and Microsoft SQL Server2005 serves as the database management system and represents data service.

Thin-client was chosen over fat-client due to the following reasons (Lowber, 2002): (1) lower Total Cost of Ownership (TOC), (2) Ease of management, (3) Lower software maintenance and distribution and (4) cheaper hardware.

The BSE Teleconsultation system helps the user to communicate and consult a trained BSE trainer via internet anytime and at any place. Studies have proven that teleconsultation system reduces transportation costs and medical fees for hospital visits (Mair and Whitten, 2000).

Furthermore, the teleconsultation system can provide the user with the accurate guideline in performing BSE and it can be one of the approaches that we can use for disseminating breast cancer information and BSE techniques. The BSE Teleconsultation system is not only designed for teleconsultation purposes, it is also designed to provide information regarding breast cancer and its prevention as well as web community services (for instances, community chat, forum/discussion board), where users can share their thoughts and knowledge. There is also a breast cancer survivor section in the BSE Teleconsultation system.

Development Process (Technique and Methods): This system uses the Rapid Prototyping Lifecycle with Versioning (Spiral Model) for the development planning and scheduling. In rapid prototyping the BSE Teleconsultation system prototypes are developed which can be quickly replaced or changed in line with testing feedback from user. The development of the BSE Teleconsultation prototype of the future system can be very helpful (El-Mousa et al., 2008), allowing users to visualize the system and provide feedback on it. Thus it can be used to clarify user requirements options.

The user requirements that were gathered during the preliminary study which specified the content of the BSE Teleconsultation system are then translated into two specific requirements: (1) Functional requirement and (2) Non-functional requirement.

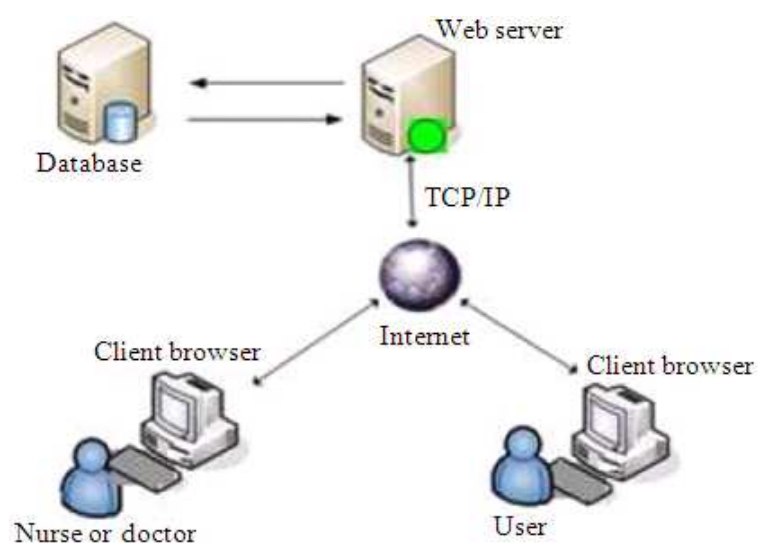

Fig. 2: Design architecture of the system

The functional requirements will specify what user wants the system to perform while the non-functional requirement is such as technical specifications in terms of hardware and software.

Modeling notation: In conjunction of using the Object Oriented Analysis and Design (OOAD) development method, the Unified Modeling Language (UML) is used. The UML is the standard language most appropriate for specifying, visualizing, constructing and documenting all the progress of developing a software system (Watson, 2010).

Coding standard: This project will be developed using the Active Server Page.NET (ASP.NET). ASP.NET is known for its wide array of functionality and suitability for developing any web-based system (Erlingsson et al., 2007). ASP. Net allows the usage of Visual Basic.Net (VB) for event handler as well as some web parts controls.

System architecture: Figure 2 illustrates the design architecture for BSE Teleconsultation system which is based on client-server model. In this model, the application processing is provided by multiple tiers; (1) Database Server, (2) Application Server and (3) PC Workstation. Database Server is where the data retrieval and updating is done while the application server or web server serves for application services and logic.

Details of the BSE Teleconsultation System GUI: Presented in this section is some of the screenshots of the BSE Teleconsultation system GUI.

Main Page: Illustrated in Fig. 3 is the main (index) page of the web-based BSE Teleconsultation system. 


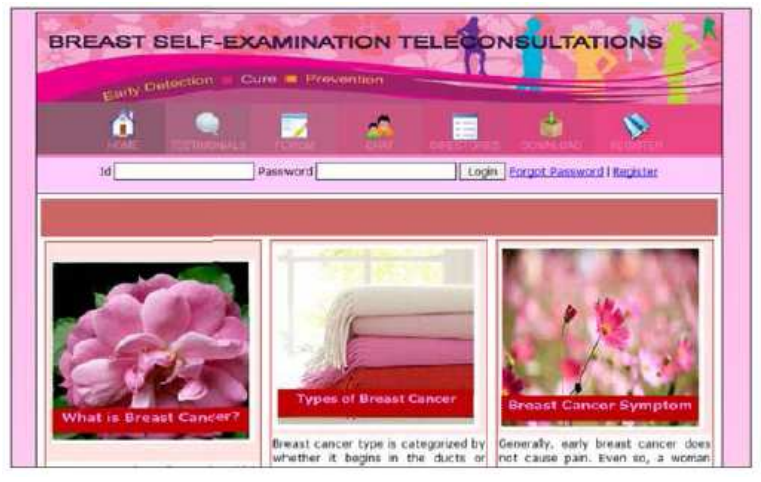

Fig. 3: The main page of the system

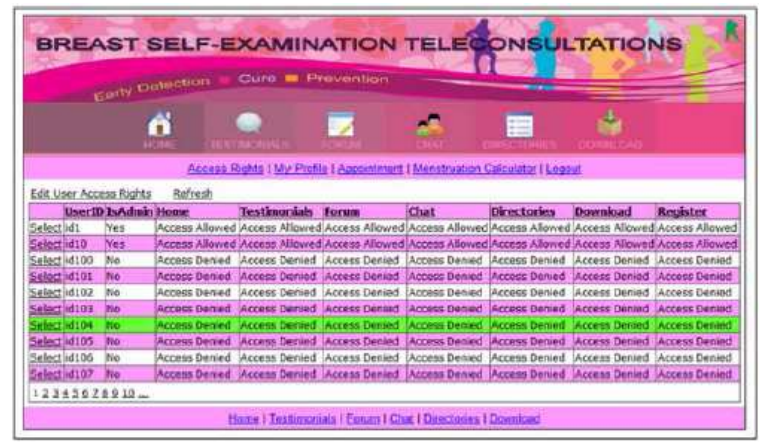

Fig. 4: The user access control panel for administrator

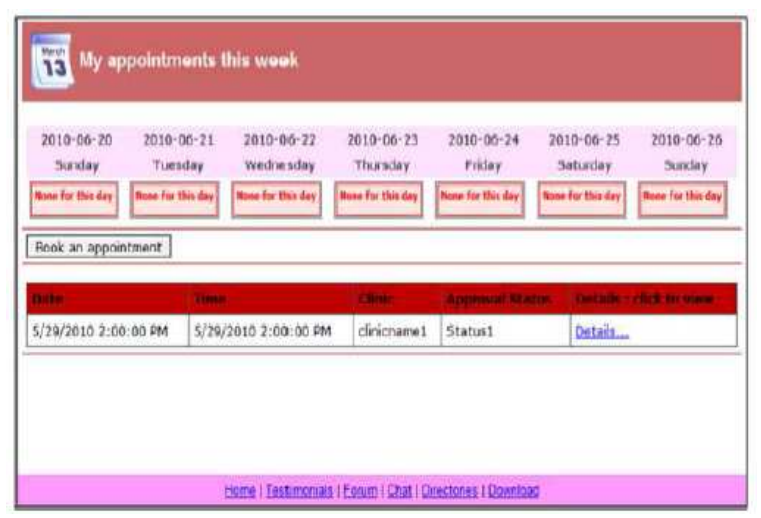

Fig. 5: The user access control panel for administrator

User access control panel: Illustrated in Fig. 4 is the access control panel for the administrator to control access in the system based on user type.

Appointment panel: Illustrate in Fig. 5 is the panel for displaying the appointment that has been booked by the user. This is user-specific panel which personalized to each user.

Chat panel: Illustrated in Fig. 6 is the chat panel which enables communication among users.

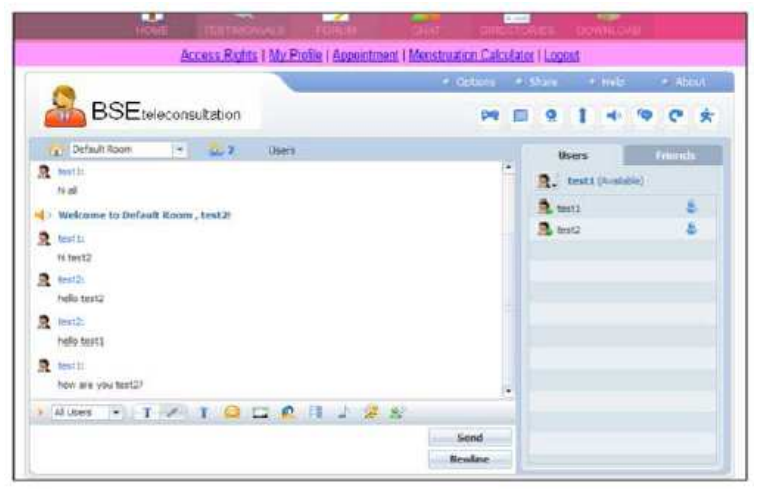

Fig. 6: The chat panel

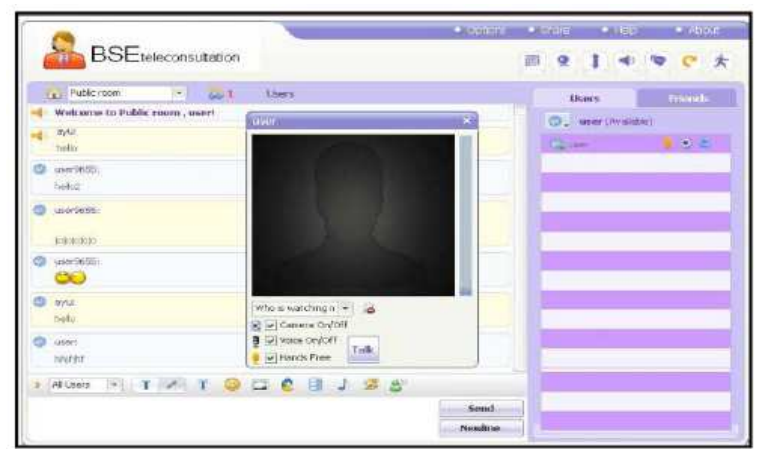

Fig. 7: Teleconsultation interface

Teleconsultation panel: Illustrated in Fig. 7 is the teleconsultation panel which enables one-to-one video chat between user and BSE educator.

\section{DISCUSSION}

This study introduced a modified UCC (MUCC) approach for incorporating user input in developing ehealth system. UCC approach has been widely applied in Social Networking Sites (SNS) but has not yet been applied in e-health domain. This study confirms that original UCC does not fit e-health system/site environment due to the following issues identified by Chin (2006): (1) contents overlapping or duplicate, (2) credibility issues of the suggested content, (3) questionable or biased content and (4) the difficulty in organizing the contents since it's structure-free.

Due to these issues, we have modified the original UCC approach suggested by Chin (2006) to overcome the abovementioned problems and to fit its application to web-based health-related system since the credibility and integrity issues are at stake (Ahern et al., 2006).

MUCC approach is differing from the original UCC approach since it is applied during the system 
development process whereas in original UCC, the suggested content is specifically for already implemented sites/system. Therefore, the modified UCC can be defined as the content and functionality of health-related sites/system suggested by user that is peer-reviewed and verified by system developer based on its relevance during the system development process.

This study confirms the significant roles of user participation using the MUCC approach in developing an e-health system/site. However, this study also has some limitations. Future research may strive to obtain responses from large sample of users. In addition, this research is conducted among small group of public users and a single nation (Malaysia). Wide range of participants might yield different results.

\section{CONCLUSION}

This study described the user's role within the development process of a Web-based BSE Teleconsultation System for disseminating breast cancer related information and BSE techniques using the Modified UCC approach. Within the development process of this solution, we used qualitative method to elicit user requirements and the system content during a development process composed by two phases carried out in iterative form: qualitative (interview), design and codification phases. The first phase (interview) represented the early efforts, where the problem was delimited and understood and the basic data was gathered for later development in the iterative cycles. In this phase and in order to increase understanding about the users' suggested content, we used open-ended interviews. A set of data to support the construction of the BSE Teleconsultation system applied the MUCC approach. In this study, user participation in MUCC approach has proven aided in designing a system based on their input.

\section{ACKNOWLEDGMENT}

The researchers would like to thank Institute of Postgraduate Studies (IPS), Universiti Sains Malaysia (USM) for their Fellowship Programme 2009/2010 and Student Ambassador Programme 2009/2010 and USM for granting the research grant entitled "Teleconsultation for Early Breast Cancer Screening for Malaysian Women". Special acknowledgement also goes to the research participants for making this study possible.

\section{REFERENCES}

Ahern, D.K., J.M. Kreslake and J.M. Phalen, 2006. What is eHealth (6): Perspectives on the evolution of eHealth research. J. Med. Internet Res., 8. DOI: 10.2196/jmir.8.1.e4

El-Mousa, A.H., Z.J. Muhsin and M.A. Al-Taee, 2008. A Web-Based Rapid Prototyping Workflow Management Information System for Computer Repair and Maintenance. J. Comput. Sci., 4: 991998. DOI: 10.3844/jcssp.2008.991.998.

Bogdan, R. and S.K. Biklen, 1998. Qualitative Research for Education: An Introduction to Theory and Methods. Needham Heights, MA: Allyn and Bacon. ISBN: 9780205482931

Burback, R.L., 1998. Three Tiered Client/Server. http://infolab.stanford.edu/ burback/dadl/node107.html

Chin, P. 2006. The value of user-generated contents part I//II. Intranet Journal, July 2007. http://www.intranetjournal.com/articles/200603/ij_ 03_07_06a.html

Davis, F.D., 1993. User acceptance of information technology: System characteristics, user perceptions and behavioral impacts. Int. J. ManMach. Stud., 38: 475-487.

deepblue.lib.umich.edu/bitstream/2027.42/30954/1 /0000626.pdf

Erlingsson, U., B. Livshits and Y. Xie, 2007. End-toend web application security. Proceedings of the 11 th USENIX workshop on Hot topics in operating systems, San Diego, CA. May 7-9, 2005. http://www.usenix.org/events/hotos07/tech/full_stu dys/erlingsson/erlingsson.pdf

Friese, S., 2011. Using ATLAS.ti for analyzing the financial crisis data. Q. Soc. Res., 12. http://nbnresolving.de/urn:nbn:de:0114fqs 1101397

Hjorth, L., 2009. Web U2: Emerging online communities and gendered intimacy in the Asiapacific region. Knowl., Technol. Policy, 22: 117-124. http://www.springerlink.com/index/275498R8K33 53742.pdf

Hughes, M., 2009. Progressive user adoption. from http://uxmatters.com/mt/archives/2009/03/progress ive-user-adoption.php

Kim, W. and O. Jeong, 2009. On social e-learning. In M. Spaniol et al (Eds.), ICWL 2009, LNCS 5686 (pp. 12-24). DOI: 10.1007/978-3-642-03426-8_2

Lannin, D.R. and T. Ponn, 2005. What should we teach women about breast self-examination today? J. Women's Health, 14: 529-530. DOI: 10.1089/jwh.2005.14.529

Lee, S.-H. and K.-H. Jo, 2009. A novel user created message application service design for bidirectional TPEG. Proceedings of the 5th International Conference on Emerging Intelligent Computing Technology and Applications. Berlin, Heidelberg: Springer-Verlag. ISBN: 3642040195 
Lee, W., C.R. Babu, J. Lee and W. Woo, 2007. VR@Home: An immersive contents creation system for 3D user-generated contents. Proceedings of the 2nd International Conference on Technologies for E-Learning and Digital Entertainment (Edutainment 2007), pp. 81-91. ISBN: 9783540730101

Lowber, P., 2002. Gartner research decision framework, DF-14-2800 white study on ThinClient vs. Fat-Client TCO.

http://h20202.www2.hp.com/Hpsub/downloads/W hiteStudy_Gartner_ThinClient_TCO.pdf

Maarop, N., T.W. Khin, S. Singh and M. Masrom, 2009. Teleconsultation service utilization key issues in the context of Malaysia: An organizational perspective. In Hussin et al. (Eds.) Proceedings of the International Conference on Research and Innovation in Information Systems 2009 (pp. 81-86). Malaysia: UTM. ISBN: 9789835206177

Mair, F. and P. Whitten, 2000. Systematic review of studies of patient satisfaction with telemedicine. British Med. J., 320: 1517-1520. DOI: 10.1136/bmj.320.7248.1517

Morris, M.G. and A. Dillon, 1997. How user perception influence software use. IEEE Software, 14: 58-65. DOI: $10.1109 / 52.595956$

No, H.S. and D.W. Rhee, 2010. A study on the development of mobile based SNS-UCC writing tool. Proceedings of the 9th International Conference on Entertainment Computing, Berlin, Heidelberg: Springer-Verlag, pp: 428-430. ISBN: 3642153984.

Ryu, M-H., S. Kim and E. Lee, 2009. Understanding the factors affecting online erderly user's participation in video UCC services. Comput. Hum. Behav., 25: 619-632. DOI: 10.1016/j.chb.2008.08.013
Schaper, L.K. and G.P. Pervan, 2007. ICT and OTs: A model of information and communication technology acceptance and utilization by occupational therapists. Int. J. Med. Inform., 76: 212-221. DOI: 10.1016/j.ijmedinf.2006.05.028

Seals, C.D., W. Moses, J. Nyagwencha, A. Martin and K. Clanton et al., 2008. Life Long Learning: Seniors in Second Life Continuum. J. Comput. Sci., 4: 1064-1070. DOI: 10.3844/jcssp.2008.1064.1070

Talib, N.A., N. Zakaria and S. Ramadass, 2008. Teleconsultations in Breast Self-Examination (BSE) practice: Alternative solution for early detection of Breast Cancer. Proceedings of the International Symposium on Information Technology (ITSim 2008), Kuala Lumpur. DOI: 10.1109/ITSIM.2008.4631600

Talib, N.A., N. Zakaria, S. Ramadass and F. Damanhoori, 2009. A review on TAM-IDT model of technology acceptance for Breast SelfExamination (BSE) teleconsultations. In Hussin et al. (Eds.) Proceedings of the International Conference on Research and Innovation in Information Systems 2009 (pp. 69-74). Malaysia: UTM. ISBN: 9789835206177

Voida, A.M., E.D. Mynatt, T. Erickson and W.A. Kellogg, 2004. Interviewing Over Instant Messaging. Vienna, Austria: ACM. pp: 1344-1347. DOI: $10.1145 / 985921.986060$

Watson, A., 2010. Visual modeling: Past, present and future. http://www.uml.org/Visual_Modeling.pdf 